\title{
Algorithm development for finding the minimum level of noise immunity of an onboard electrical complex during control tests
}

\author{
Vladimir Kozlovsky ${ }^{1 *}$, Pavel Nikolaev ${ }^{2}$, Alexander Podgorny ${ }^{1}$, Alexander Saksonov ${ }^{1}$ and \\ Luiza Shamina ${ }^{3}$ \\ ${ }^{1}$ Samara State Technical University, 443001, Samara, Russia \\ ${ }^{2}$ PJSC AVTOVAZ, 445024 Togliatti, Russia \\ 3 National Technology Initiative Center for Advanced Manufacturing Technologies based on the \\ Institute of Advanced Manufacturing Technologies of Peter the Great St. Petersburg Polytechnic \\ University Polytechnicheskaya, 29, St.Petersburg, 195251, Russia
}

\begin{abstract}
An algorithm for finding the minimum level of noise immunity of the onboard electrical complex during control tests is developed in this paper. The expression is obtained for the frequency tuning step of a narrow-band electromagnetic action and an algorithm is developed that compared with the standard to more accurately find the minimum level of noise immunity of ETS.
\end{abstract}

Keywords: automobile, electrical on-board complex, electromagnetic compatibility.

\section{Introduction}

The existing requirements for the frequency adjustment step are not sufficient for a full assessment of electrotechnical systems (ETS) and on-Board electrical complex (BEC) of motor vehicles (MV) in the parameters of electromagnetic compatibility (EMC). In this case, it is possible to skip ranges with low noise immunity, and therefore inaccurately define minimum characteristics of noise immunity. This means that there are certain risks associated with their safe operation. Another problem is a long time to find the minimum noise immunity of ETS (BEC). Because, even if the frequency range is determined, in which there are malfunctions, then one has to look for the levels of noise immunity at each frequency.

EMC problems are known to be occurred due to the parasitic resonant properties of electrical circuits. Hence the problem of more accurate and fast finding the minimum level of noise immunity of an electrical system is solved using the section of resonant circuits of the general theory of electrical circuits. The most difficult case should be considered when it is necessary to identify a frequency range with low noise immunity formed by one

*Corresponding author: kozlovsky_vn@mail.ru 
resonant circuit, because it is more difficult to find it than the range formed by several circuits.

\section{The main part}

Let's accept the condition: the passbands of the parasitic resonant circuits do not overlap. The bandwidth of the resonant circuit has to be greater than the frequency tuning step of the narrow-band action.

The resonant frequency of a circuit and its bandwidth are related by the expression:

$$
\mathrm{Q}=\frac{f_{p}}{\Delta f}
$$

where $\mathrm{Q}$ - a figure of merit; $f_{p}$ - resonant frequency of the oscillating circuit; $\Delta f-$ bandwidth equal to

$$
\Delta f=f u-f l
$$

where $f_{l}$ and $f_{u}$ are lower and upper frequencies of the loop bandwidth.

Since

$$
f_{u}-f_{p}=f_{p}-f_{l}
$$

the expression (3) can be written as:

$$
f_{p}=\frac{2 Q f_{l}}{2 Q-1}
$$

The electromagnetic effect begins with the frequency $f_{1}$. If we assume that it coincides with the lower frequency of the first resonant circuit $f_{l 1}$, i.e.

$$
f 1=f l 1
$$

then the optimal frequency adjustment step should be such that the second frequency of the impact is resonant. According to this condition, the bandwidth of parasitic resonant circuits does not overlap. The noise immunity characteristic is complex and depends on many MV parameters. If EMC problems are detected during exposure, this range can be investigated in detail at any given step. But if there are no malfunctions, we assume that the second frequency of exposure falls on the lower frequency of the bandwidth of the second resonance

$$
f 2=f l 2=f p 1
$$

Hence, the first step is, considering (3)

$$
h_{1}=f_{p 1}-f_{l 1}=f_{l 2}-f_{l 1}=\left(\frac{2 Q f_{1}}{2 Q-1}-f_{1}\right)=f_{1}\left(\frac{1}{2 Q-1}\right)
$$

Based on the arguments given above, let us write

$$
f_{2}=f_{1}+f_{1}\left(\frac{1}{2 Q-1}\right)=f_{1}\left(\frac{2 Q-1+1}{2 Q-1}\right)=f_{1}\left(\frac{2 Q}{2 Q-1}\right)
$$

The second step of the impact adjustment is equal to

$$
h_{2}=\frac{f_{2}}{2 Q-1}=f_{1}\left(\frac{2 Q}{(2 Q-1)^{2}}\right)
$$

Similarly

$$
f_{3}=f_{2}+f_{1}\left(\frac{2 Q}{(2 Q-1)^{2}}\right)=f_{1}\left(\frac{2 Q}{2 Q-1}+\frac{2 Q}{(2 Q-1)^{2}}\right)
$$

Accordingly, the third step will be equal to 


$$
\begin{aligned}
& h_{3}=\frac{f_{3}}{(2 Q-1)}=f_{1}\left(\frac{2 Q}{2 Q-1}+\frac{2 Q}{(2 Q-1)^{2}}\right) \times \frac{1}{(2 Q-1)}=(11) \\
& =f_{1}\left(\frac{2 Q}{(2 Q-1)^{2}}+\frac{2 Q}{(2 Q-1)^{3}}\right)
\end{aligned}
$$

The fourth frequency of impact

$$
\begin{aligned}
& f_{4}=f_{3}+h_{3}=f_{1}\left(\frac{2 Q}{(2 Q-1)^{2}}\right)= \\
& =f_{1}\left(\frac{2 Q}{2 Q-1}+\frac{2 Q}{(2 Q-1)^{2}}\right)+f_{1}\left(\frac{2 Q}{(2 Q-1)^{2}}+\frac{2 Q}{(2 Q-1)^{3}}\right)= \\
& =f_{1}\left(\frac{2 Q}{2 Q-1}+\frac{4 Q}{(2 Q-1)^{2}}+\frac{2 Q}{(2 Q-1)^{3}}\right)
\end{aligned}
$$

Formulas (6) - (12) show that the frequency adjustment is a geometric progression with the parameter $\left(\frac{2 Q}{2 Q-1}\right)$. From this

$$
f_{n}=f_{1}\left(\frac{2 Q}{2 Q-1}\right)^{n-1}
$$

where $\mathrm{n}=1,2,3 \ldots$

Accordingly $f_{n} \in\left\lfloor f_{\min } ; f_{\text {max }}\right\rfloor$, where $f_{\text {min }}$ and $f_{\max }$ are the minimum and maximum frequencies of the range in which the ETS (BEC) tests are performed.

From the experimental data presented below in the fourth chapter, the maximum figure of merit is found, which is 33.8. Then expression (13) takes the form

$$
f_{n}=1,015^{n-1} f_{1}
$$

Expressions (13) and (14) are derived for the case when the frequency of exposure coincides with the resonance.

The figure of merit parameter is defined either from the results of calculating the mathematical model of the tested ETS (BEC) or is selected from the statistical analysis of previously obtained experimental data of similar systems.

In practice, the frequency range in which there is a malfunction ETS (BEC) is formed by multiple resonant circuits and wider than the frequency adjustment step to an electromagnetic field.

The following algorithm is used for finding the minimum level of noise immunity of an electrical system. At the first iteration, the electrotechnical system installed in MV with the specified $E_{e f 1}$ is tested for electromagnetic effect. Frequency tuning is performed according to (14). There is a malfunction of the electrical system in this frequency range $\Delta F_{1}$. If

$$
\Delta F_{1}>f_{n+2}-f_{n}
$$

where

$$
\Delta F_{l}=\left\{f_{n} ; f_{n+1} ; f_{n+2}\right\}
$$

then at the second iteration, the level is reduced to the value of $E_{e f 2}$. Tests are carried out in the range $\Delta F_{l}$ with this exposure level $E_{e f 2}$. Based on their results, the frequency range of $\Delta F_{2}$ is found. By analogy with (15), it is compared with the adjustment step of narrow-band electromagnetic influence.

Iterations continue to reduce the exposure level until the condition is met

$$
\Delta F_{j}=f_{n+2, j}-f_{n, j}
$$

where $\Delta F_{j}$ is the frequency range in which the electrical system is disrupted at the level of $E_{\text {efj }}$;

$j=1,2,3 \ldots$ is the number of the electromagnetic effect level; 
and

$$
\begin{gathered}
\Delta F_{j}=\left\{f_{n, j} ; f_{n+1, j} ; f_{n+2, j}\right\}, \\
\Delta F_{j} \subset \Delta F_{1} .
\end{gathered}
$$

The case $j=1$ is a special one where iterations to reduce the exposure level are not performed. Changing the levels of subsequent impacts is made from the condition

$$
E_{e f j+1}=k E_{e f j}
$$

where $k$ is the coefficient selected from the condition

$$
0,71 \leq \mathrm{k}<1 \text {. }
$$

The coefficient $k$ has the following physical meaning: the specified reduction in the level of electromagnetic influence should not be greater than the ratio of the induced interference at the resonance frequency to the induced interference at the extreme frequencies of the bandwidth of this resonant circuit.

If condition (16) is met, the minimum level of noise immunity is at the frequency

$$
f_{\text {min }}=\frac{f_{n+2, j}+f_{n, j}}{2}
$$

and equal to

$$
E_{\min }=E\left(f_{\min }\right)
$$

The proposed approach is explained in Figure 1, and the algorithm for finding the minimum level of noise immunity of an electrical system when testing for narrow-band effects is shown in Figure 2.

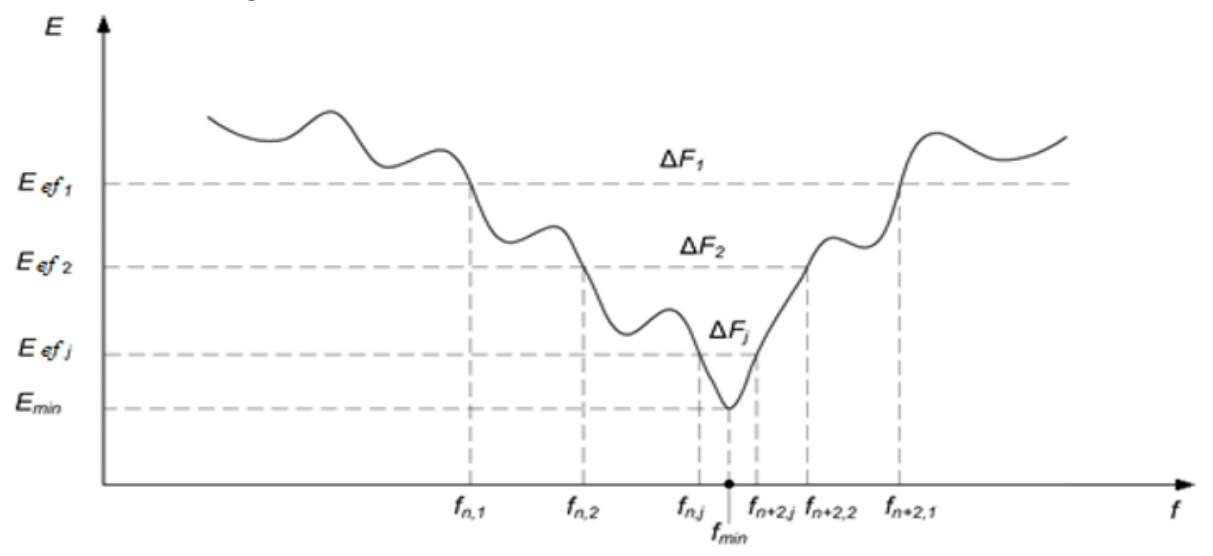

Fig. 1. Finding the minimum level of noise immunity of an electrical system

Experimental studies of the electromechanical power steering of a car of a well-known Russian brand using the proposed algorithm have shown that the accuracy of finding the minimum level of noise immunity increases in comparison with the standard algorithm (Fig. 3). In standard tests, the minimum level of noise immunity was $71 \mathrm{~V} / \mathrm{m}$, while the found level is $63 \mathrm{~V} / \mathrm{m}$ using the proposed approach. The accuracy can be calculated using the known relative error formula

$$
\delta=\frac{\left|E_{t r}+E_{m}\right|}{E_{m}} \times 100 \%
$$


where $E_{t r}$ is the true level of noise immunity found by the proposed algorithm; $E_{m}$ is the measured level of noise stability found by the standard algorithm.

Hence

$$
\delta=\frac{|63 \mathrm{~V} / \mathrm{m}-71 \mathrm{~V} / \mathrm{m}|}{63 \mathrm{~V} / \mathrm{m}} \times 100 \%=12,7 \%
$$

As seen from the estimation, the accuracy with the application of the proposed algorithm increases by more than $12,7 \%$.

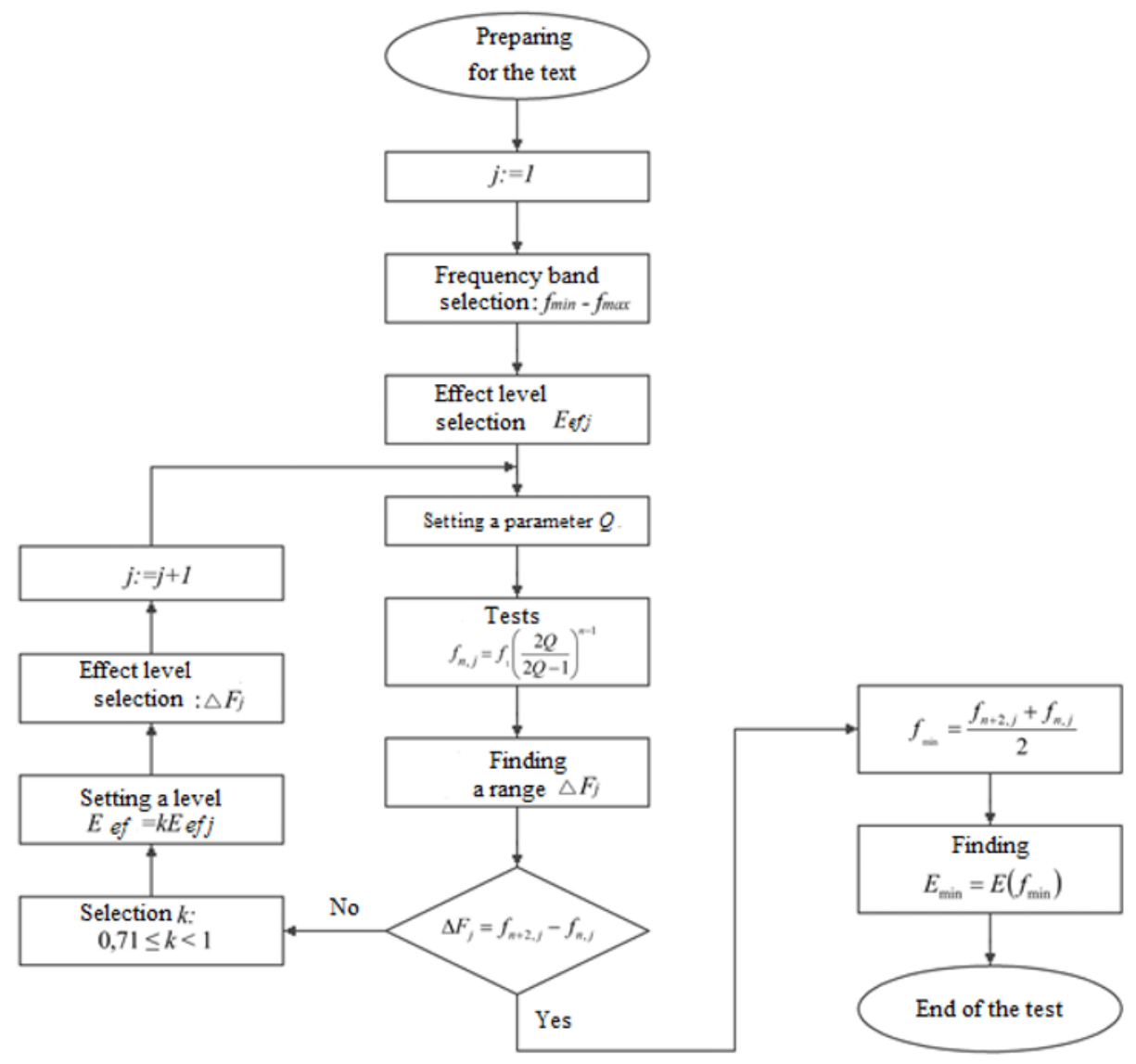

Fig. 2. Algorithm for finding the minimum level of noise immunity of MV (BEC) during narrowband impact tests 


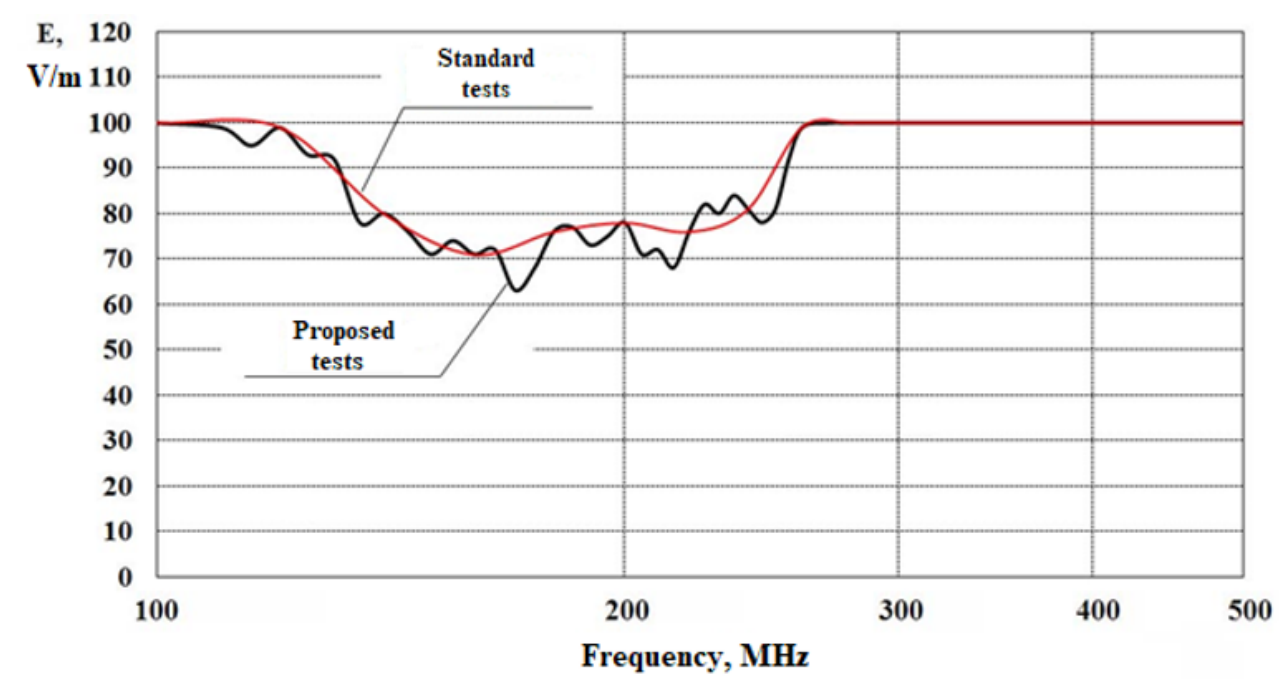

Fig. 3. Noise immunity of an electromechanical power steering of a car

\section{Conclusion}

The expression is obtained for the frequency tuning step of a narrow-band electromagnetic action and an algorithm is developed that compared with the standard to more accurately find the minimum level of noise immunity of ETS. The research results in the accuracy of finding the level of noise immunity increases by $12.7 \%$.

\section{Acknowledgment}

The work was carried out with the financial support of the grant of the President of the Russian Federation NSh-2515.2020.8

\section{References}

1. Antonova, V., Alekseev, S., Tarasov, A., Scheglova, N., Klyavin, O., \& Borovkov, A. (2019). Analysis and use of SIMP method in optimization of a car hood design. Paper presented at the E3S Web of Conferences, , 140 doi:10.1051/e3sconf/201914004017

2. Borisenko, V., Kiselev, A., Leoro, L., Borovkov, A., \& Boldyrev, Y. (2019). K\&C suspension parameters stability by production tolerances. Paper presented at the E3S Web of Conferences, , 140 doi:10.1051/e3sconf/201914007007

3. Ivanov, A., Abyzov, O., Galyshev, Y., \& Rumyantsev, V. (2020). The performance test of the car catalytic converter in the conditions of urban operation. Paper presented at the E3S Web of Conferences, , 164 doi:10.1051/e3sconf/202016403018

4. Klochkov, Y., Gazizulina, A., \& Golovin, N. (2016). Assessment of organization development speed based on the analysis of standards efficiency. Paper presented at the Proceedings - 2nd International Symposium on Stochastic Models in Reliability Engineering, Life Science, and Operations Management, SMRLO 2016, 530-532. doi:10.1109/SMRLO.2016.93

5. Korovkin, N., \& Diop, C. Y. (2016). Minimization of electromagnetic fields intensity and optimization of electrical wiring networks in healthcare facilities. Paper presented 
at the Proceedings of the 2016 IEEE North West Russia Section Young Researchers in Electrical and Electronic Engineering Conference, EIConRusNW 2016, 604-607. doi:10.1109/EIConRusNW.2016.7448255

6. Kozlovsky, V., Klochkov, Y., Ostapenko, M., Ushanova, N., \& Antipov, D. (2016). Conformity assessment of car quality databases exemplified on the case of the russian car manufacturer 'auto VAZ'. Paper presented at the 2016 5th International Conference on Reliability, Infocom Technologies and Optimization, ICRITO 2016: Trends and Future Directions, 57-60. doi:10.1109/ICRITO.2016.7784925

7. Kozlovsky, V. N., Lysov, V. E., Ermakov, V. V., Antipov, D. V., \& Skripnuk, D. F. (2019). Calculation and statistical experiment on the monte carlo method when assessing the stability of the technical characteristics of the automobile generator set in mass production. Paper presented at the Proceedings of the 2019 IEEE Conference of Russian Young Researchers in Electrical and Electronic Engineering, ElConRus 2019, 565-568. doi:10.1109/EIConRus.2019.8657034

8. Papirovskiy, A., Alekseev, S., Tarasov, A., \& Borovkov, A. (2019). Development of numerical methods and virtual tests for cyclic durability by finite element method. Materials Physics and Mechanics, 42(6), 859-869. doi:10.18720/MPM.4262019_17

9. Rakov, V., Ivanov, V. N., Karpov, Y. G., Melehin, V. F., Izmailov, R. A., Yun, V. K., \& Zaripova, D. A. (2019). Method for determining the basic energy characteristics of elements of a hybrid car engine. Paper presented at the IOP Conference Series: Earth and Environmental Science, , 337(1) doi:10.1088/1755-1315/337/1/012066

10. Zeziulin, D. V., Yu Tyugin, D., Shashkina, K. M., Kuzmichev, V. A., \& Beresnev, P. O. (2018). Autonomous electric commercial vehicle for difficult operating conditions. Paper presented at the IOP Conference Series: Materials Science and Engineering, , 386(1) doi:10.1088/1757-899X/386/1/012003 